\title{
INCLUSIÓN EDUCATIVA SEGÚN EL ALUMNADO Y PROFESORADO: INTEGRACIÓN DE INMIGRANTES Y GÉNERO
}

\author{
Laura Sánchez-Pujalte \\ Universidad Internacional de Valencia \\ marialaura.sanchez@campusviu.es \\ Talía Gómez Yepes \\ Universidad Internacional de Valencia \\ talia.gomez@campusviu.es \\ Diego Navarro \\ Universidad Internacional de Valencia \\ diego.navarrom@campusviu.es \\ Edgardo Etchezahar \\ Universidad Internacional de Valencia \\ edgardo.etchezahar@campusviu.es
}

Recepción Artículo: 17 mayo 2021 Admisión Evaluación: 17 mayo 2021

Informe Evaluador 1: 18 mayo 2021

Informe Evaluador 2: 22 mayo 2021

Aprobación Publicación: 02 junio 2021

\section{RESUMEN}

Cada año aumenta de forma ostensible la cantidad de población migrante a nivel global que intenta acceder al sistema educativo, encontrándose con múltiples dificultades para su integración, incluyendo la integración en materia de género. Diferentes estudios han analizado el grado de integración de la población migrante en el ámbito educativo, así como la aceptación por parte de la comunidad educativa receptora. También se ha estudiado el rechazo a trabajar asignaturas vinculadas al género y la diversidad, lo que deja al descubierto que las demandas de organizaciones como Ia UNESCO o la OMS, sobre la necesidad de avanzar en un modelo educativo que parta del principio de igualdad, no han sido escuchadas. No obstante, no han sido desarrollados estudios que analicen ambos aspectos en el ámbito educativo, contrastando la perspectiva de dos de sus principales actores: el alumnado y el profesorado. Participaron en el estudio 491 personas adultas argentinas ( $58 \%$ mujeres y $42 \%$ hombres), de los cuáles 312 eran estudiantes universitarios y 179 profesores, con edades comprendidas entre los 18 y los 75 años. Se usó un cuestionario auto aplicable, que indagaba las actitudes hacia la inclusión de inmigrantes y de la perspectiva de género en el ámbito educativo, además de variables socio-demográficas. Los resultados evidenciaron que el alumnado presentó actitudes más favorables tanto hacia la inclusión de inmigrantes en el ámbito educativo, 


\section{INCLUSIÓN EDUCATIVA SEGÚN EL ALUMNADO Y PROFESORADO: INTEGRACIÓN DE INMIGRANTES Y GÉNERO}

así como hacia la diversidad de género en educación, en comparación con los profesores que formaron parte del estudio. Así también, las mujeres mostraron actitudes significativamente más favorables hacia la inclusión de los inmigrantes en el ámbito educativo, como también una mayor perspectiva favorable con respecto al género, en comparación con los hombres. Se discuten los alcances y límites del estudio, así como el rol del profesorado y alumnado en la inclusión educativa.

Palabras clave: Inclusión educativa; inmigrantes; género; profesorado; alumnado

\section{ABSTRACT}

Educational inclusion according to students and teachers: immigrants' integration and gender. Every year, the amount of global migrant population that tries to enter the educational system increases dramatically, encountering multiple difficulties in their integration. Likewise, gender integration is another of the pending debts. Different studies have analyzed the degree of integration of the migrant population in the educational field, as well as the acceptance by the receiving educational community. The refusal to work on subjects related to gender and diversity has also been studied, which reveals that the demands of organizations such as UNESCO or WHO, regarding the need to advance in an educational model that starts from the principle of equality, have not been heard. However, no studies have been developed that analyze both aspects in the educational field, contrasting the perspective of two of its main actors: the student body and the teaching staff. 491 Argentine adults participated in the study ( $58 \%$ women and $42 \%$ men), of the results 312 were university students and 179 teachers, aged between 18 and 75 years. A self-administered questionnaire was used, which investigated attitudes towards the inclusion of immigrants and the gender perspective in education, as well as sociodemographic variables. The results showed that the students presented more favorable attitudes both towards the inclusion of immigrants in the educational field, as well as towards gender diversity in education, compared to the teachers who were part of the study. Likewise, women carry out activities significantly more favorable towards the inclusion of immigrants in the educational field, such as a greater favorable perspective with respect to gender, compared to men. The scope and limits of the study are discussed, as well as the role of teachers and students in educational inclusion.

Keywords: educational inclusion; immigrants; gender; professors; students

\section{INTRODUCCIÓN}

Los movimientos globales de personas a través de las fronteras han aumentado drásticamente en las últimas décadas, lo que supone un reto para los Estados nacionales a la hora de mantener la cohesión social dentro de una población cada vez más diversa (Sauer, 2019; Yuval-Davis, 2011). Este aumento mundial del número de inmigrantes sugiere la necesidad de examinar las prácticas de las instituciones encargadas de su reasentamiento en los países de acogida (Castles, 2003; Plancarte Cansino, 2017). Como consecuencia, cada año aumenta la cantidad de población migrante a nivel global que intenta ingresar al sistema educativo, encontrándose con múltiples dificultades para su integración (Thomazet, 2009). Al respecto es importante analizar el papel que cumplen las instituciones educativas en el proceso de integración de los y las migrantes, así como del éxito de las mismas (Marfleet, 2006). Asimismo, la integración en materia de género es otra de las deudas pendientes del sistema educativo (Sauer, 2019). Por ejemplo, se ha estudiado cómo muchas veces desde diferentes ámbitos educativos se rechaza trabajar asignaturas vinculadas al género y diversidad (Yuval-Davis, 2011), lo que deja al descubierto que las demandas de organizaciones como la UNESCO, sobre la necesidad de avanzar en un modelo educativo que parta del principio de igualdad, no han sido escuchadas (Martínez-Usarralde, 2021).

Diferentes estudios han analizado el grado de integración de población migrante en el ámbito 
educativo, así como la aceptación por parte de la comunidad educativa receptora (Christie y Sidhu, 2002). También múltiples estudios dejan al descubierto que las creencias de los y las profesionales de la educación y del alumnado en formación ante la inclusión educativa de la población migrante e igualdad de género podrían estar influenciadas por la formación inicial que reciben en cuanto a inclusión, igualdad, género, atención a la diversidad, ciudadanía, entre otras (Boer et al., 2011). Otras investigaciones dejan a la vista cómo el conocimiento escolar y sus supuestos y atribuciones en función del género desempeñan un papel clave en el mantenimiento de las jerarquías y desigualdades de género en la sociedad (Arnot y Pinson, 2005), perpetuando las injusticias a través de las desigualdades educativas basadas en características individuales y grupales como el género, la etnia, la lengua, la religión, entre otras. Aspectos que exigen un debate más agudo y políticamente consciente sobre la adecuación de la educación que se imparte (Preston, 2009) y el reconocimiento de que los enfoques de la educación son múltiples, fragmentados, contradictorios, pudiendo no tener los mismos niveles de eficacias para todos y todas. Las instituciones educativas tienen un papel fundamental en la integración de la población infantil y adolescente, ya que facilitan la transición a la ciudadanía y robustecen el sentido de pertenencia identitaria (Cassity y Gow, 2005). Sin embargo, gran parte de la investigación en este campo pone de manifiesto los problemas y las limitaciones en la prestación de apoyo educativo y para la inclusión al alumnado migrante (Yuval-Davis, 2011). La mayoría de los investigadores coinciden en dos aspectos a la hora de hablar de inclusión. En primer lugar, la inclusión no se refiere únicamente al acceso a la institución educativa (Marfleet, 2006). En segundo lugar, también se coincide en que la inclusión es un concepto difícil de definir y que tiene muchas acepciones (Artiles et al., 2006), por lo cual desde hace mucho tiempo, la inclusión es un concepto central en la investigación sobre necesidades educativas especiales y atención a la diversidad en lo que respecta a género, que sustituye a conceptos precursores, como el de integración. Por lo tanto, es importante revisar aspectos conceptuales sobre la educación inclusiva y considerarla como una línea fundamental sobre la que los sistemas escolares, las escuelas y las aulas deberían lograr, y como tal, una expresión de una filosofía educativa (Martínez-Usarralde, 2021).

La inclusión educativa se puede definir como una respuesta educativa a la diversidad presente, desde una perspectiva positiva, que promueve la participación de todo el alumnado y comunidad educativa teniendo en cuenta sus logros y desde la supresión de barreras de todos los agentes educativos (Plancarte Cansino, 2017). En esta línea, la inclusión educativa promueve la igualdad de oportunidades desde el horizonte de logro académico y social (Yuval-Davis, 2011), reconociendo e integrando las diferencias socioculturales y de capacidades. Un enfoque basado en la inclusión educativa implica, por parte del profesorado, la adopción de una posición favorable hacia los derechos humanos, que en las instituciones educativas debe responder a las necesidades y realidades del alumnado migrante (Dyson et al., 2002). Asimismo, el profesorado y los centros educativos vertebrarán sus intervenciones de acuerdo al principio de igualdad de oportunidades, sin segregar a ninguna persona como consecuencia de su discapacidad, dificultad de aprendizaje, género o pertenencia a cualquier minoría o grupo social, entre otras (Cagney, 2009). De esta forma, la inclusión es un proceso para abordar y responder a la diversidad de necesidades de todo el alumnado mediante una mayor participación en el aprendizaje, las actividades culturales y comunitarias, y reduciendo la exclusión desde la educación (Boer et al., 2011; UNESCO, 2015). Dicho proceso implicará, por tanto, transformaciones educativas en el contenido, enfoque, estructura y estrategias del profesorado, con una visión común que cubre a todo el alumnado y una convicción sobre la responsabilidad ética y social de las instituciones en esta materia de inclusión, igualdad de género y por ende construcción de ciudadanía (Martínez-Usarralde, 2021). 


\section{INCLUSIÓN EDUCATIVA SEGÚN EL ALUMNADO Y PROFESORADO: INTEGRACIÓN DE INMIGRANTES Y GÉNERO}

A nivel mundial, contamos con marcos que consagran los derechos de los niños y las niñas a tener una educación libre de discriminación y que responda a sus necesidades educativas y culturales especiales (Dyson et al., 2002). El marco de la Educación para Todos de Dakar, la Declaración de los Derechos Humanos de la ONU de 1949 y la Convención sobre los Derechos del Niño de 1989, afirman el derecho de los niños y las niñas a una educación de calidad que reconozca la diversidad y que no discrimine por motivos de género, discapacidad, origen nacional o de sus padres. Sin embargo, estas y otras declaraciones de derechos han tenido impacto limitado y han acabado teniendo una función meramente simbólica en diferentes contextos como es el argentino (Christie y Sidhu 2002).

\section{OBJETIVO DE LA INVESTIGACIÓN}

El objetivo principal de este estudio fue analizar las diferencias en el grado de acuerdo con creencias sobre a la inclusión educativa de la población migrante y la igualdad de género, en una muestra de estudiantes universitarios y profesorado argentino.

\section{MÉTODO \\ Participantes}

Participaron en el estudio 491 personas adultas residentes en Argentina (58\% mujeres y $42 \%$ hombres), de los cuáles 312 eran estudiantes universitarios y 179 profesores, con edades comprendidas entre los 18 y los 75 años $(M=46,02 ; D T=10,87)$. Con respecto a la edad de cada grupo, el alumnado $(M=29,95 ; D T=10,46)$ y profesores $(M=50,51 ; D T=11,26)$.

\section{Instrumentos}

Para la recolección de datos se utilizó un cuestionario autoadministrable, que indagaba una serie de creencias sobre la inclusión de inmigrantes en el ámbito educativo y sobre la igualdad de género, además de variables socio-demográficas.

Creencias hacia la Inclusión Educativa de los Inmigrantes: Se evaluaron siete ítems que contienen creencias relacionadas con la inclusión educativa de los inmigrantes ( $=.79$ ) (ver Tabla 1). Los aspectos específicamente evaluados tienen en cuenta diversos indicadores propuestos por Booth y Ainscow (2002), centrándose en el rol que el gobierno y las instituciones educativas deben asumir en la inclusión y atención a la diversidad del alumnado migrante. El formato de respuesta fue tipo Likert con cinco anclajes en grados de acuerdo. Mayores puntuaciones indican creencias más favorables hacia la inclusión en la educación (a excepción del ítem 7 que se encuentra invertido).

Creencias sobre la Desigualdad de Género en el ámbito Educativo: Se evaluaron ocho ítems que contienen creencias relacionadas con la inclusión educativa de género ( $=.83$ ) (ver Tabla 2). Los aspectos específicamente evaluados tienen en cuenta diversos indicadores propuestos por Glick y Fiske (2001) y Johannesen-Schmidt y Eagly (2002), centrándose en la igualdad de género, aplicada a diferentes instancias del sistema educativo: "El acceso a contenidos sobre igualdad de género es un derecho educativo"; "Las instituciones educativas deberían visibilizar las desigualdades de género como una construcción socio-cultural"; y "El alumnado debería participar activamente en la elaboración de contenidos educativos relacionados a la igualdad de género". El formato de respuesta fue tipo Likert con cinco anclajes en grados de acuerdo. Mayores puntuaciones indican creencias más favorables hacia la inclusión en la educación (a excepción de los ítems 1 y 5 que son invertidos).

Datos sociodemográficos: Se indagaron variables como el género y la edad de las personas participantes del estudio. 


\section{Procedimiento y análisis de datos}

La muestra se conformó de forma intencional, siendo las personas que formaron parte del estudio invitadas a participar de la investigación de manera voluntaria. Se les solicitó el consentimiento informado y se les informó a los y las participantes que los datos derivados de esta investigación se utilizarían exclusivamente con fines académicos y de difusión científica. Para realizar el análisis de Ios datos se utilizó el programa estadístico SPSS 24.

\section{RESULTADOS}

En primer lugar, se procedió a analizar las creencias sobre la inclusión educativa de los inmigrantes, comparando los resultados del alumnado y profesorado que formó parte de este estudio (Tabla 1).

Tabla 1.

Creencias sobre la inclusión educativa según profesores y alumnado

\begin{tabular}{|c|c|c|c|c|}
\hline & Alumnado & Profesorado & $t(p)$ & $d$ de Cohen \\
\hline $\begin{array}{l}\text { 1. Las universidades deberían garantizar } \\
\text { el acceso de estudiantes inmigrantes. }\end{array}$ & $\begin{array}{l}4.18 \\
(1.27)\end{array}$ & $\begin{array}{l}3.88 \\
(1.32)\end{array}$ & $\begin{array}{l}2.442 \\
(.015)\end{array}$ & .23 \\
\hline $\begin{array}{l}\text { 2. El gobierno debe garantizar que los hijos } \\
\text { de inmigrantes reciban la educación } \\
\text { necesaria. }\end{array}$ & $\begin{array}{c}4.30 \\
(1.19)\end{array}$ & $\begin{array}{c}4.35 \\
(1.06)\end{array}$ & $\begin{array}{l}-.468 \\
(.640)\end{array}$ & .04 \\
\hline $\begin{array}{l}\text { 3. En las escuelas donde hay niños y niñas } \\
\text { inmigrantes, se debería fomentar el } \\
\text { estudio de la realidad de esos países. }\end{array}$ & $\begin{array}{l}3.80 \\
(1.23)\end{array}$ & $\begin{array}{l}3.51 \\
(1.38)\end{array}$ & $\begin{array}{l}2.401 \\
(.017)\end{array}$ & .22 \\
\hline $\begin{array}{l}\text { 4. El Estado debería aumentar la cantidad } \\
\text { de profesores inmigrantes en las escuelas } \\
\text { públicas. }\end{array}$ & $\begin{array}{l}3.41 \\
(1.24)\end{array}$ & $\begin{array}{l}3,01 \\
(1.28)\end{array}$ & $\begin{array}{l}3.453 \\
(.001)\end{array}$ & .31 \\
\hline $\begin{array}{l}\text { 5. El sistema educativo debe fomentar } \\
\text { valores cívicos orientados al } \\
\text { reconocimiento de la cultura y etnias de } \\
\text { poblaciones migrantes. }\end{array}$ & $\begin{array}{c}4.19 \\
(1.16)\end{array}$ & $\begin{array}{l}4.05 \\
(1.24)\end{array}$ & $\begin{array}{l}1.273 \\
(.204)\end{array}$ & .11 \\
\hline $\begin{array}{l}\text { 6. La mayoría del alumnado inmigrante } \\
\text { ocupa espacios de la educación pública, } \\
\text { empeorando la calidad de esta }\end{array}$ & $\begin{array}{c}1.59 \\
(1.09)\end{array}$ & $1.45(.88)$ & $\begin{array}{l}1.473 \\
(.141)\end{array}$ & .14 \\
\hline $\begin{array}{l}\text { 7. El alumnado inmigrante debe integrarse } \\
\text { en la dinámica escolar del resto, ya que su } \\
\text { presencia es un hecho accidental }\end{array}$ & $\begin{array}{l}2.21 \\
(1.37)\end{array}$ & $\begin{array}{l}2.86 \\
(1.36)\end{array}$ & $\begin{array}{l}-4.938 \\
(.001)\end{array}$ & .47 \\
\hline
\end{tabular}

Nota. En cursiva los ítems en los que se hallaron diferencias estadísticamente significativas. Fuente: Elaboración propia. 


\section{INCLUSIÓN EDUCATIVA SEGÚN EL ALUMNADO Y PROFESORADO: INTEGRACIÓN DE INMIGRANTES Y GÉNERO}

Como se puede observar en la Tabla 1, se hallaron diferencias estadísticamente significativas en cuatro de las siete creencias sobre la inclusión educativa de los inmigrantes. En todos los casos, se observó que el alumnado obtuvo niveles más altos que el profesorado en cuanto a las creencias acerca de la inclusión educativa de los inmigrantes. Se destaca el ítem número siete que, si bien obtuvo una media más alta el profesorado con respecto al alumnado, en este caso el ítem es invertido (la presencia del inmigrante en el contexto escolar es "accidental").

Por otra parte, se testeó en qué medida los profesores y el estudiantado estaban de acuerdo con diferentes creencias sobre la igualdad de género en el ámbito educativo. A continuación, en la Tabla 2 , se presentan una serie de afirmaciones sobre la temática y los puntajes medios del alumnado y del profesorado.

Tabla 2.

Creencias sobre la inclusión educativa según profesores y alumnado

\begin{tabular}{|c|c|c|c|c|}
\hline & Alumnado & Profesorado & $t(p)$ & $d$ de Cohen \\
\hline $\begin{array}{l}\text { 1. Son los padres quienes deben decidir si } \\
\text { sus hijos acceden a contenidos educativos } \\
\text { sobre igualdad de género }\end{array}$ & $\begin{array}{c}2.05 \\
(1.49)\end{array}$ & $\begin{array}{c}2.39 \\
(1.63)\end{array}$ & $\begin{array}{r}-2.277 \\
(.023)\end{array}$ & .21 \\
\hline $\begin{array}{l}\text { 2. El alumnado debería participar } \\
\text { activamente en la elaboración de } \\
\text { contenidos educativos relacionados a la } \\
\text { igualdad de género }\end{array}$ & $\begin{array}{c}3.70 \\
(1.36)\end{array}$ & $\begin{array}{c}3.00 \\
(1.46)\end{array}$ & $\begin{array}{l}5.143 \\
(.001)\end{array}$ & .49 \\
\hline $\begin{array}{l}\text { 3. Las instituciones educativas deberían } \\
\text { visibilizar las desigualdades de género } \\
\text { como una construcción socio-cultural }\end{array}$ & $\begin{array}{c}3.97 \\
(1.36)\end{array}$ & $\begin{array}{c}3.78 \\
(1.52)\end{array}$ & $\begin{array}{l}1.408 \\
(.160)\end{array}$ & .13 \\
\hline $\begin{array}{l}\text { 4. El acceso a contenidos sobre igualdad de } \\
\text { género es un derecho educativo }\end{array}$ & $\begin{array}{c}4.39 \\
(1.18)\end{array}$ & $\begin{array}{c}4,21 \\
(1.34)\end{array}$ & $\begin{array}{l}1.518 \\
(.130)\end{array}$ & .14 \\
\hline $\begin{array}{l}\text { 5. El género determina una parte relevante } \\
\text { de nuestros comportamientos }\end{array}$ & $\begin{array}{l}2.68 \\
(1.43)\end{array}$ & $\begin{array}{c}3.02 \\
(1.52)\end{array}$ & $\begin{array}{l}-2.358 \\
(.019)\end{array}$ & .23 \\
\hline $\begin{array}{l}\text { 6. Todas la personas tienen derecho a } \\
\text { reclamar por una mayor igualdad de } \\
\text { género en cualquier momento de su vida }\end{array}$ & $\begin{array}{c}4,26 \\
(1.16)\end{array}$ & $\begin{array}{c}3,90 \\
(1.44)\end{array}$ & $\begin{array}{l}2.928 \\
(.004)\end{array}$ & .27 \\
\hline $\begin{array}{l}\text { 7. Es necesario proteger los derechos de } \\
\text { las mujeres mediante acciones de igualdad } \\
\text { de género en el ámbito educativo }\end{array}$ & $\begin{array}{c}4,49 \\
(1.01)\end{array}$ & $\begin{array}{l}4,26 \\
(1.15)\end{array}$ & $\begin{array}{l}2.204 \\
(.028)\end{array}$ & .21 \\
\hline $\begin{array}{l}\text { 8. En todas las escuelas se debería enseñar } \\
\text { sobre las desigualdades de género }\end{array}$ & $\begin{array}{l}4,74 \\
(.76)\end{array}$ & $4,57(.96)$ & $\begin{array}{l}2.006 \\
(.045)\end{array}$ & .19 \\
\hline
\end{tabular}

Nota. En cursiva los ítems en los que se hallaron diferencias estadísticamente significativas Fuente: Elaboración propia. 
Posteriormente, se procedió a agrupar todos los ítems (por un lado los de inclusión educativa de los inmigrantes y, por otro, los relativos al género y educación), con la finalidad de observar si se hallaban diferencias a nivele general entre alumnado y profesorado. Con respecto al puntaje total agrupando los ítems de inclusión educativa de inmigrantes $(t(489)=2.640 ; p<.01 ; d$ de Cohen $=$ .26), se observan diferencias significativas entre el alumnado $(M=4,01 ; D T=.93)$ y el profesorado $(M=3,78 ; D T=.83)$, siendo el alumnado quien presenta creencias más inclusivas. Asimismo, con respecto a las creencias sobre inclusión educativa con respecto al género, también se observaron diferencias significativas $(t(489)=2.640 ; p<.01 ; d$ de Cohen $=.27)$ entre alumnado $(M=4,27 ; D T$ $=.87)$ y profesorado $(M=4,02 ; D T=.94)$, siendo el alumnado quien presenta creencias más igualitarias.

Por último, se analizó si había diferencias significativas en las creencias del alumnado y profesorado según el género y edad de los participantes del estudio. Con respecto al alumnado, se observaron diferencias en cuanto a las creencias sobre la inclusión de inmigrantes en el ámbito educativo $(t(304)=3.478 ; p<.001 ; d$ de Cohen $=.50)$, siendo las participantes mujeres $(M=4,09 ; D T=$ .86) quienes obtuvieron mayores puntajes, en comparación con los hombres $(M=3,59 ; D T=1.11)$ al igual que sucede con las creencias sobre la igualdad de género y educación $(t(279)=3.374 ; p<$ . 001; $d$ de Cohen $=.48)$, donde también las mujeres $(M=4,35 ; D T=.78)$ obtuvieron medias significativamente más altas en comparación con los hombres $(M=3,88 ; D T=1.11)$. En lo referente a la edad del alumnado, las relaciones con las creencias acerca de la inclusión educativa de inmigrantes $(r=-.185 ; p<.001)$ y de género $(r=-.206 ; p<.001)$ resultaron significativas y negativas (es decir, a mayor edad, menos creencias a favor de la inclusión de inmigrantes y sobre la igualdad de género), pero con fuerza baja.

En cuanto al profesorado, se repite -en parte- lo observado para el alumnado: se presentan diferencias en cuanto a las creencias sobre la inclusión de inmigrantes en el ámbito educativo ( $t$ (176) $=2.062 ; p<.05 ; d$ de Cohen $=.34)$, siendo las participantes mujeres $(M=3,88 ; D T=.80)$ quienes obtuvieron mayores puntajes, en comparación con los hombres $(M=3,60 ; D T=.81)$, a diferencia de lo que sucede con las creencias sobre la igualdad de género y educación, en donde no se hallaron diferencias estadísticamente significativas. En lo referente a la edad del profesorado, no se hallaron diferencias con respecto a las creencias acerca de la inclusión educativa de inmigrantes, pero sí con las creencias acerca de la igualdad de género $(r=-.161 ; p<.001)$, las que resultaron significativas y negativas (es decir, a mayor edad, menos creencias a favor de la igualdad de género), pero con fuerza baja.

\section{DISCUSIÓN Y CONCLUSIONES}

Hoy en día, las diferencias individuales en la configuración del sistema educativo son muy importantes (Farris, 2017). Es necesario proporcionar condiciones adecuadas para los individuos en términos de necesidades sociales, personales y educativas, independientemente de su origen. La educación inclusiva es un enfoque para atender a los niños y niñas en entornos de educación general y también apoyar y acoger la diversidad entre todo el alumnado, eliminando la exclusión social, la desigualdad de género, además de atender sus diferencias y necesidades individuales (UNESCO, 2015).

Desde la Agenda 2030, en países como Argentina se plantea la necesidad de mejorar la calidad de la educación en sus diferentes niveles, así como la mejora y actualización de la formación de los y las docentes de cara a la promoción de la inclusión educativa y la igualdad de oportunidades en el contexto educativo (UNESCO, 2015). El presente estudio evidencia que el tipo y calidad de la formación docente genera creencias y emociones diferentes en el profesorado y el alumnado universitario. Por ello, desde las instituciones educativas argentinas se han de considerar las preocupacio- 


\section{INCLUSIÓN EDUCATIVA SEGÚN EL ALUMNADO Y PROFESORADO: INTEGRACIÓN DE INMIGRANTES Y GÉNERO}

nes que desde diferentes estamentos y organizaciones a nivel mundial se planifiquen para mejorar en este contexto el proceso enseñanza-aprendizaje desde la inclusión y la igualdad (Dyson et al., 2002).

En nuestro estudio se observó que, en lo que refiere a las creencias hacia la Inclusión Educativa de los Inmigrantes, existen diferencias significativas entre el profesorado y el alumnado. Particularmente al manifestar creencias y expectativas acerca de lo que es y debería ser la inclusión educativa, la atención a la diversidad y la igualdad de género de población escolar inmigrante, donde el alumnado valora de forma significativamente más positiva las prácticas inclusivas y de igualdad en comparación con los profesores. Esto podría deberse a la brecha generacional y formación recibida por un grupo u otro. Al respecto, estudios llevados a cabo por Martínez-Usarralde (2021) y Preston (2009) demuestran que la diferencia generacional y la calidad de la educación recibida podrían llevar tanto a educadores como a educandos a manifestar creencias y expectativas diferenciadas acerca de lo que debería ser la inclusión educativa, la atención a la diversidad y la igualdad de género. Por ello, en la medida en que cada vez más la comunidad educativa y científica cuenta con mayor conocimiento y herramientas para abordar estas problemáticas, se plantea como imprescindible abrir espacios de comunicación, formación y planificación por parte de las instituciones educativas, con la finalidad de dar mayor formación al profesorado en lo que a inclusión y genero refiere, con el objetivo de eliminar a mediano plazo las creencias erróneas que pueden estar afectando los procesos de inclusión e igualdad en el ámbito escolar en el contexto argentino (Farris, 2017).

Se analizó también si había diferencias significativas en las creencias del profesorado según el género y edad de los participantes del estudio. Las profesoras obtuvieron puntuaciones significativamente más altas que los profesores en lo que refiere a creencias sobre la inclusión educativa de la población inmigrante. De acuerdo a Arnot y Pinson (2005), esto podría deberse a lo que el autor Ilama "El factor de crecimiento social", el cual apunta a que las mujeres podrían mostrar creencias más positivas hacia los aspectos sociales de la inclusión, debido a que suelen tener mayor formación en educación inclusiva. Al respecto, diversos estudios han intentado determinar de qué manera las creencias de los profesores con o sin educación inclusiva hacia las prácticas de educación inclusiva, afectan a tales procesos y como se pueden alcanzar avances en pro de la población escolar migrante (Dyson et al., 2002). En la mayoría de estos estudios se ha llegado a la conclusión de que el hecho de tener cursos de educación inclusiva condujo a cambios positivos en las actitudes de los profesores o futuros profesores sobre la educación inclusiva (UNESC0, 2015).

Por otra parte, no sucede lo mismo con las creencias sobre la igualdad de género y educación, en donde no se hallaron diferencias estadísticamente significativas. Lo que se ve sustentado por investigaciones llevadas a cabo por autores como Martínez-Usarralde (2021) y Cagney (2009), quienes encontraron que no había diferencia estadísticamente significativa entre las actitudes de los profesores, hombres y mujeres, hacia la inclusión en términos de género.

Nuestro estudio tiene varias limitaciones, ya que la muestra con la que se trabajó no es representativa y, por lo tanto, los resultados informados no pueden extrapolarse al resto de alumnado y profesorado. Sin embargo, constituye un primer informe que da lugar a próximos desarrollos, en los que se sugiere tener en cuenta estos aspectos. Sin lugar a dudas, es necesario seguir trabajando en la formación de los futuros docentes en lo que respecta a políticas y prácticas de inclusión e igualdad y con los docentes en programas de actualización y sensibilización ante la problemática social y personal que genera la exclusión y la desigualdad a corto, mediano y largo plazo (YuvalDavis, 2011). 


\section{REFERENCIAS BIBLIOGRÁFICAS}

Arnot, M. y Pinson, H. (2005). The education of asylum-seeker and refugee children: A study of LEA and school values, policies and practices. Faculty of Education, University of Cambridge.

Artiles, A. J., Kozleski, E., Dorn, S. y Christensen, C. (2006). Learning in Inclusive Education Research: Re-mediating Theory and Methods with a Transformative Agenda. Review of Research in Education 30. 65-108. doi:10.3102/0091732X030001065.

Boer, A., Pijl, S. J. y Minnaert, A. (2011). Regular primary school teachers' attitudes towards inclusive education: a review of the literature. International Journal of Inclusive Education, 15(3), 331-353.

Booth, T. y Ainscow. M. (2002). Index for inclusion (2nd ED). Developing leaning and participation in schools ( $\left.2^{a} e d\right)$. CSIE.

Cagney, T. L. (2009) Attitudes of general education teachers toward including students with special needs, Master Of Science, lowa State University.

Cassity, E. y Gow, G. (2005). Shifting space and cultural place: The transition experiences of African young people in west Sydney schools. Trabajo presentado en la Australian Association of Educational Research, Annual Conference, Noviembre 27 - Diciembre 1, Sydney.

Castles, S. (2003). Towards a Sociology of Forced Migration and Social Transformation. Sociology, 37(1), 3-34.

Christie, P., y Sidhu. R. (2002). Responding to refugees: Globalisation and the challenges facing Australian schools. Mots Pluriel 21 (Mayo).

Dyson, A., Howes, A. y Roberts, B. (2002). A Systematic Review of the Effectiveness of School-level Actions for Promoting Participation by all Students. In Research Evidence in Education Library. EPPI-Centre, Social Science Research Unit.

Johannesen-Schmidt, M. C. y Eagly, A. H. (2002). Another Look at Sex Differences in Preferred Mate Characteristics: The Effects of Endorsing the Traditional Female Gender Role. Psychology of Women Quarterly, 26(4), 322-328. doi:10.1111/1471-6402.t01-2-00071

Farris, S. (2017). In the name of women's rights. The rise of femonationalism. Durham. Duke University Press.

Glick, P. y Fiske, S. T. (2001). Ambivalent sexism. Advances in Experimental Social Psychology, 115188. doi:10.1016/s0065-

Marfleet, P. (2006). Refugees in a global era. New York: Palgrave Macmillan.

Martínez-Usarralde, M. J. (2021). Inclusión educativa comparada en UNESCO y OCDE desde la cartografía social. Educación XX1, 24(1), 93-115, http://doi.org/10.5944/educXX1.26444

Plancarte Cansino, P. A. (2017). Inclusión educativa y cultura inclusiva. Revista de Educación Inclusiva, 10(2), 213-226.

Preston, R. (2009). What do and might the GMRs achieve? How might we know? Trabajo presentado en el seminario Reaching and Teaching the Most Marginalised? Critical Seminar on the UNESCO Global Monitoring Report 2010.

Thomazet, S. (2009). From Integration to Inclusive Education: Does Changing the Terms Improve Practice? The International Journal of Inclusive Education 13(6). 553-563.

doi:10.1080/13603110801923476.

UNESCO (2015). Replantear la educación. ¿Hacia un buen común mundial? http://unesdoc.unesco.org/images/0023/002326/232697s.pdf

Yuval-Davis, N. (2011). The politics of belonging: Intersectional contestations. Sage Publishing. 
